\title{
Enhanced M-Gear Protocol for Lifetime Enhancement in Wireless Clustering System
}

\author{
Gurpreet Kaur \\ $M$ Tech \\ Computer Science Student, \\ Sri Guru Granth \\ Sahib World University, \\ Fatehgarh Sahib, PB, India
}

\author{
Sukhpreet Kaur \\ Asst.Professor, \\ Computer Science, \\ Sri Guru Granth Sahib \\ World University, \\ Fatehgarh Sahib, PB,India
}

\begin{abstract}
Due to the presence of radio the nodes can transfer data directly to the sink node but if radio stops its working there will be a huge waste of energy. Now days, a protocol named GEAR is used for routing in WSN.GEAR is an energy efficient protocol which is used for the routing purpose in WSN. Traditionally various protocols like LEACH were proposed but very not that much efficient. After that the concept of the gateway nodes were introduced but one gateway node was unable to handle such large amount of data .The energy consumption of the network was more and the lifetime of network was less so a new approach is proposed. In this paper the advancement in the MGEAR protocol is presented from the result obtained it is concluded that the proposed algorithm is much better and efficient than the traditional algorithm. The energy consumption of the nodes present in the network is less and the life time of the network is more.
\end{abstract}

\section{Keywords}

WSN,M-GEAR, LEACH ,Gateway nodes

\section{INTRODUCTION}

WSN stands for wireless sensor network .It is a combination of multiple nodes and these nodes establish the communication between sources and sink node. The nodes included in the WSN consume an amount of energy for the purpose of transferring data during the communication process. [4] The procedure of data transferring among various nodes is also known as routing. The route selection highly effects the lifetime of the network as the energy level gets low the lifetime of the system will be decrease in same extent. Hence there are number of techniques or algorithms available which are used to increase the lifetime of the WSN and also provide the mechanism for efficient power consumption by the nodes for transferring data. [4] LEACH is a protocol which is used to increase the energy efficiency and lifetime of the system. LEACH stands for Low Energy Adaptive Clustering Hierarchy. In LEACH, all the nodes contained in a local cluster and a single node among all of the nodes behaves like a cluster-head or base station. [5] If the cluster heads were chosen a fixed throughout the system life time, as in conventional clustering algorithms, it is easy to see that the unlucky sensors chosen to be cluster-heads would die quickly, ending the useful lifetime of all nodes belonging to those clusters. Due to the presence of radio the nodes can transfer data directly to the sink node but if radio stops its working there will be a huge waste of energy. Now days, a protocol named GEAR is used for routing in WSN [3] .GEAR is an energy efficient protocol which is used for the routing purpose in WSN. [1] GEAR is Geographical and Energy Aware Routing scheme. The working of the GEAR is as follows:
First of all, it searches for the geographical position of the next neighbor node for the purpose of data transmission. And at the area where the data packets have to be delivered, a repetitive routing algorithm is used there.[2] Because there is a large amount of data which is to be delivered therefore the data is divided into small packets or sets by using an automated method. Then a process named data fusion is employed for collecting the separated data. [3] In this protocol a gateway is installed at the central location of the network to enhance the lifetime of the network. The gateway is installed to gather the data from the nearby cluster head and then transmits it further hence it increases the energy efficiency of the network

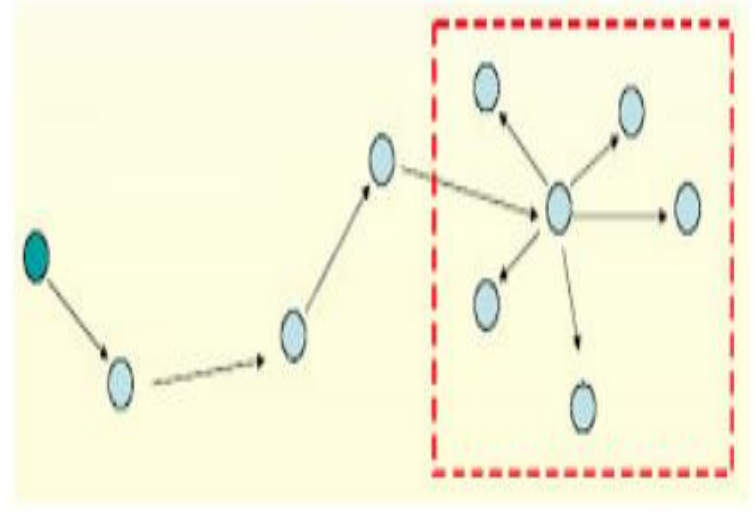

Fig 1 M-GEAR protocol [2]

\section{LIMITATION OF TRADAITIONAL APPROACHES}

Routing is the process of selecting best path for transmission of data from the source to destination. Number of routing protocols has been proposed till date for routing in wireless networks. Nodes send the data to the sink through cluster heads. The problems with the earlier designed protocols were:

In the earlier designed techniques the sink was located in the centre of the network. [3] Then, techniques were designed in which the sink was located outside the network. The communication between the sink and the far away located nodes was made difficult by locating the sink outside the network. [6]It resulted in decreased lifetime \& efficiency of the network A gateway node was introduced outside the network between the sink and the network to ease the communication process. The gateway node was unable to handle the data at larger extent. Communication was easier for the nearby located nodes but the problem was again with the 
far away located nodes. Placing a single gateway node was problem.

\section{PROPOSED WORK}

Routing protocols are designed to increase the efficiency and the lifetime of the system. Routing is done such that least amount of energy is used and the nodes remain alive for longer time while transmission of data. When the sink is located outside the network, the energy consumption of the far away located nodes increases that affected the lifetime of the network. a gateway node was introduced between the sink and the nodes. Now the data transmission was done from nodes to gateway node which further sends it to the sink. But one gateway node was unable to handle such large amount of data. To overcome this problem number of solutions is proposed.

It is proposed to increase the count of gateway nodes. Increasing the number of gateway nodes will distribute the load among them and hence will improve the lifetime of the gateway nodes which in turn will increase lifetime of the system. Gateway nodes could not be placed randomly in a network. So, Network will be divided into number of sections Each section will have its own gateway node. The nodes of that section will send the data to the gateway node which will further transmit it to the sink. Now, communication is easier for gateway nodes that are near to the sink, but the gateway nodes that are located far away will again consume larger amount of energy for transmitting data to the sink. To overcome this problem, Gateway to gateway communication is introduced. Now, the farther gateways will send data to the gateway nodes that are located near to the sink and these nearby gateway nodes will further send the data to the sink. This will improve energy efficiency and the lifetime of the network

\section{METHODOLOGY}

In this propose work the M-GEAR protocol is proposed that is considered to be efficient than the traditional protocol. The methodology of the proposed work is defined below:

1) Initially the network is creating and the various network parameters are initialized by the user. The parameters like energy, Area etc are initialized.

2) Next step after the initialization of the parameter is to divide the network into grids. The network is uniformly divided into grids.

3) After the network is divided into grid, next step is deployment of the nodes. So the nodes are deployed in the network.

4) In this gateway nodes are introduced. These nodes are used for the communication between the cluster heads and the sink

5) In this step the formation of cluster head is done from each uniform region. These cluster head will receive data from the nodes that are present in the grid and will send it to the gateway node.

6) After this the energy is dissipated in the network. the energy dissipation take place by using energy model .

7) Next step after the communication is performed is the calculation of the performance parameter. These parameters will define the performance of the system,
8) Finally the comparison is done between the traditional approach and the proposed approach. From the results it is concluded that the proposed approach is efficient and better than the traditional approach

Initialization of the network parameter .Parameters like Energy, area etc are initialized

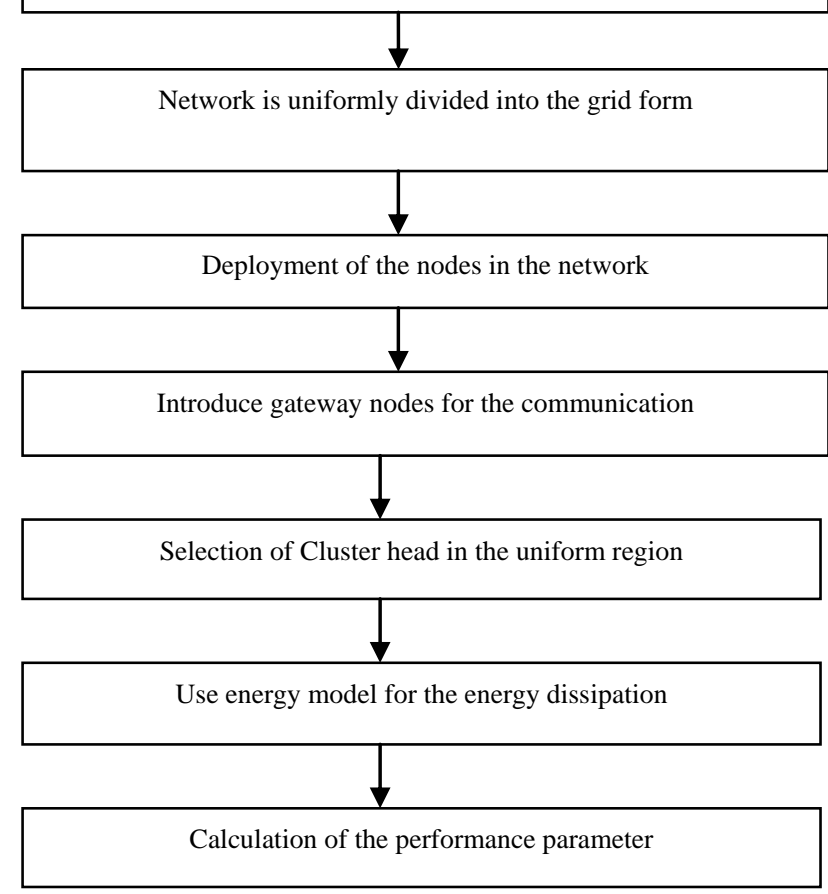

Fig 2 flow diagram of the proposed work

\section{RESULTS AND DISCUSSION}

In this section there is discussion about the results of proposed method of routing in the wireless sensor network. In this paper M-GEAR protocol is proposed. The graph given below depicts the comparisons between the proposed and the traditional algorithm. The proposed technique is considered to be efficient than the traditional as the energy utilization is less and the lifetime of the network is more.

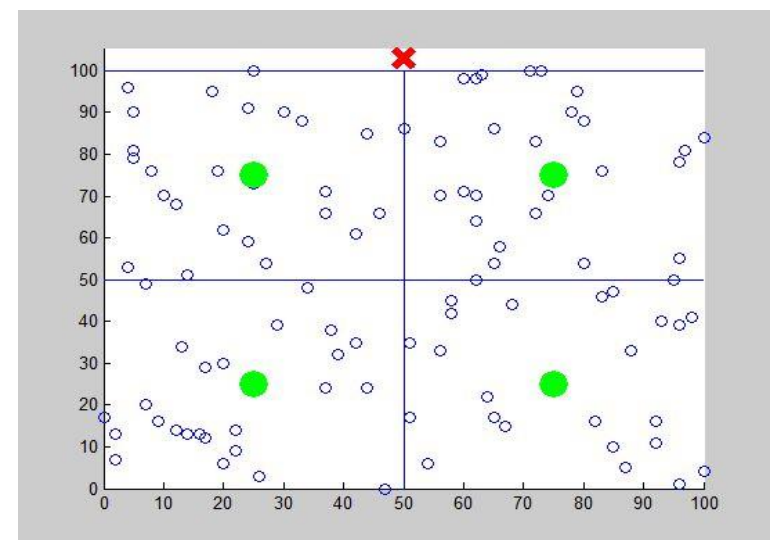

Fig 3 shows the deployment of the nodes in the network 


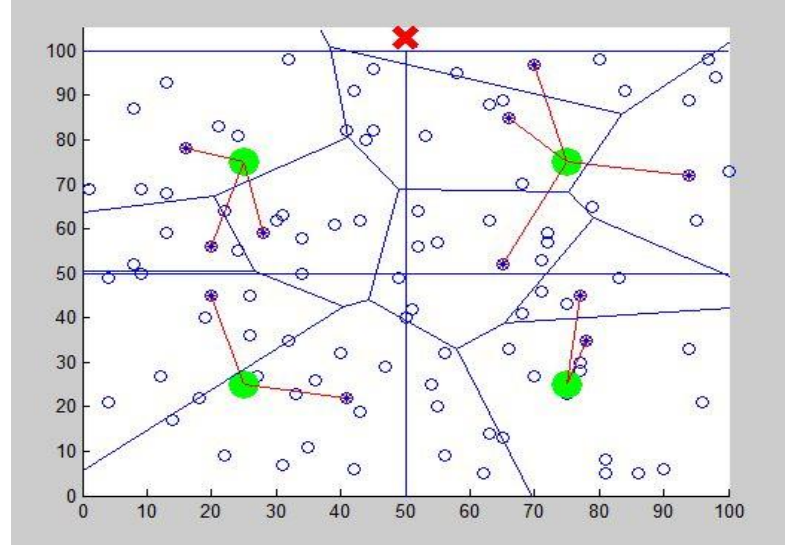

Fig 4 shows the communication from gateway nods to cluster head

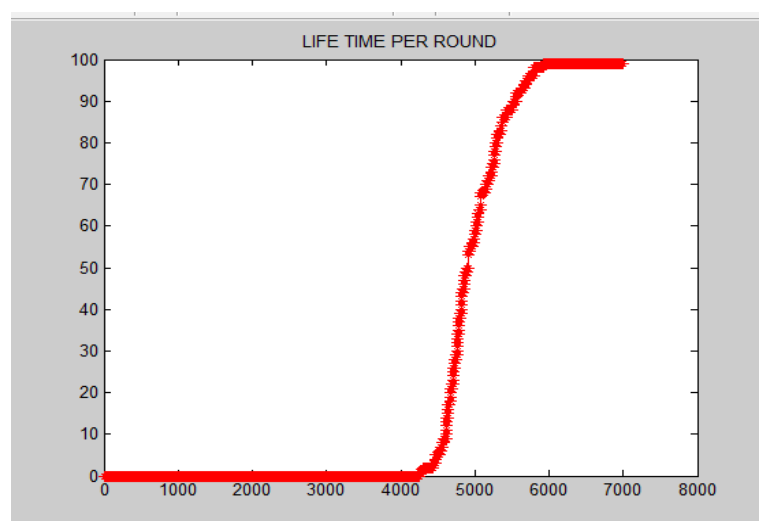

Fig 5 shows the lifetime of the node per rounds

The figure 6,7,8 and 9 shows the comparison between the proposed and the tradiational approaches .

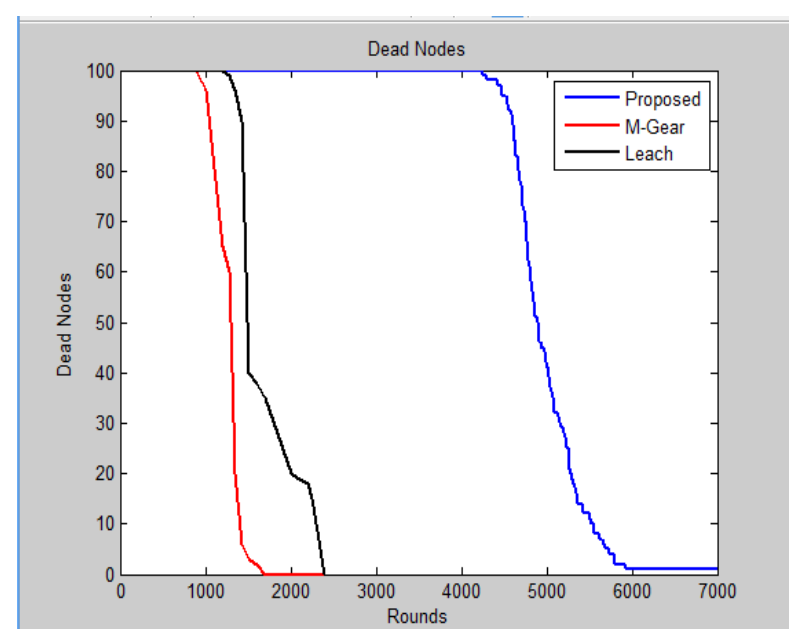

Fig 6 shows the comparison between proposed, M-Gear and Leach on the basis of the Dead nodes in the network

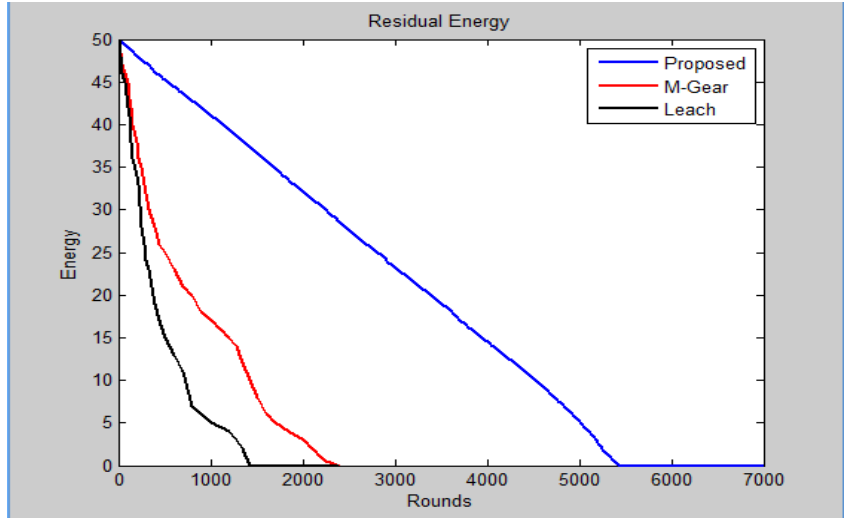

Fig 7 shows the comparison between proposed, M-Gear and Leach on the basis of the residual energy in the network

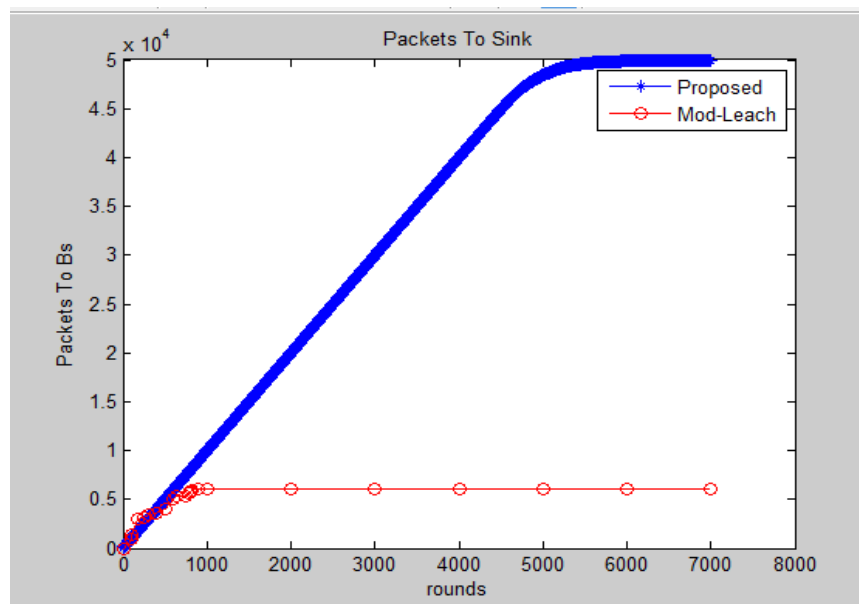

Fig 8 shows the comparison between proposed, and the Mod- Leach on the basis packet sent to the sink

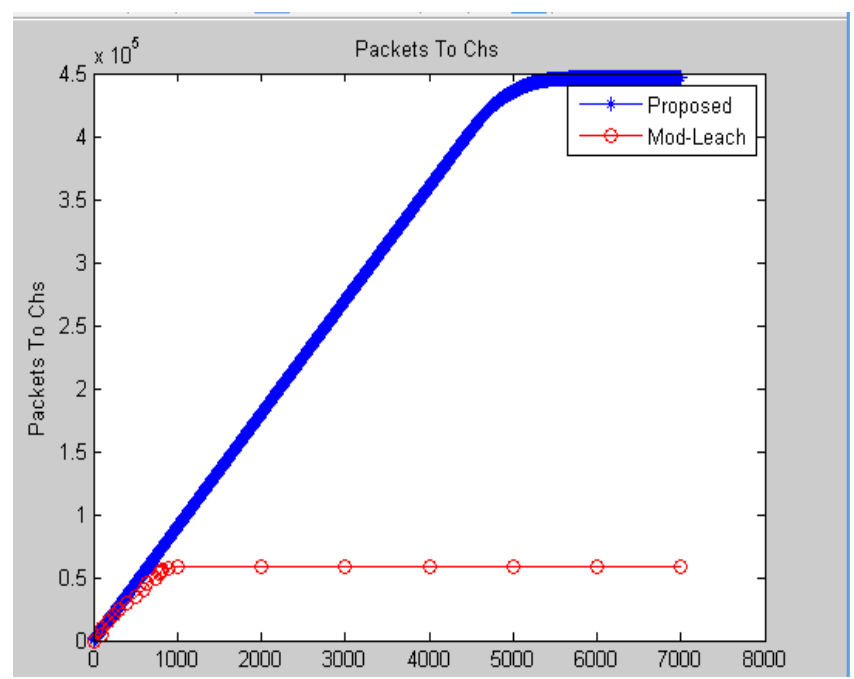

Fig 9 shows the comparison between proposed, and the Mod- Leach on the basis of packet sent to the cluster head

Table 1 shows the network parameters

\begin{tabular}{|l|c|}
\hline \multicolumn{1}{|c|}{ Parameters } & Value \\
\hline Nodes & 100 \\
\hline Network Size & $100 * 100$ \\
\hline Initial Energy (Eo) & $0.5 \mathrm{j}$ \\
\hline
\end{tabular}




\begin{tabular}{|l|c|}
\hline ETX & $5 \mathrm{nj}$ \\
\hline ERX & $5 \mathrm{nj}$ \\
\hline Efs & $1 \mathrm{pj}$ \\
\hline Emp & $0.0013 \mathrm{pj}$ \\
\hline Gateway nodes & 4 \\
\hline Sink location & $\mathrm{X}=50, \mathrm{Y}=105$ \\
\hline Rounds & 7000 \\
\hline
\end{tabular}

\section{CONCLUSION AND FUTURE SCOPE}

In this paper a protocol is proposed in which the route is selected by acquiring the information about the location of the neighbor and then the route is decided according to it. From the results obtained it is concluded that this protocol is better than the traditional routing protocols. A comparison is made between the traditional and the GEAR protocol that shows the life time of the network is increased and the energy consumption of the nodes is reduced by using this protocol.

From the results obtained it is concluded that this protocol is efficient than the traditional protocol as the network lifetime and the energy consumption of the nodes is decreased. In future this protocol can be further enhanced to increase the life time of the network. As the life time of the network is increased the network becomes more efficient

\section{REFERENCES}

[1] Yan Yu, " Geographical and Energy Aware Routing: a recursive data dissemination protocol for wireless sensor networks

[2] Q. Nadeem, "M-GEAR: Gateway-Based Energy-Aware Multi-Hop Routing Protocol for WSNs"

[3] Haixia Zhao(2013), "A New Secure Geographical Routing Protocol Based on Location Pair wise Keys in Wireless Sensor Networks", IJCSI International Journal of Computer Science Issues, Vol. 10, Issue 2, No 2,Pp 365-372

[4] Gaurav Srivastav(2013), "Effective Sensory Communication using GEAR Protocol " International Journal of Science and Research (IJSR) vol 9, issue 4, Pp 1809-1815

[5] Shamsad Parvin (2008), "Routing Protocols for Wireless Sensor Networks: A Comparative Study , International Conference on Electronics, Computer and Communication (ICECC) Pp 891-894

[6] Monica R Mundada (2012), "A study on energy efficient routing protocols in wireless sensor networks "International Journal of Distributed and Parallel Systems (IJDPS) Vol.3, No.3,Pp 311-330

[7] Rama Sundari Battula (2013) , "Geographic Routing Protocols for Wireless Sensor Networks: A "International Journal of Engineering and Innovative Technology (IJEIT) Volume 2, Issue 12,Pp 39-42

[8] Neeraj Dewli (2015), "A Comparative Analysis of MGEAR and MODLEACH Energy Efficient WSN Protocols "(IJCSIT) International Journal of Computer Science and Information Technologies, Vol. 6 (3) , pp 2641-2644

[9] Neha Rathi(2012)," A review on routing protocols for application in wireless sensor networks "International
Journal of Distributed and Parallel Systems (IJDPS) Vol.3, No.5,Pp 39-58

[10] Lovepreet Kaur(2014), “Energy-Efficient Routing Protocols in Wireless Sensor Networks: A Survey "International Journal of Computer Applications ,Volume 100- No.1,Pp 25-29

[11] Neeraj Rajgure, "Geographical Data Collection in Sensor Networks with Self-Organizing Transaction ClusterHeads"

[12] Jay G. Shah(2015), “Technical Review of Wireless Sensor Network and its Protocol International journal for research in emerging science and technology, volume-2, issue-1,Pp 35-41

[13] Shital Y. Agrawal(2013), “A Survey on Location Based Routing Protocols for Wireless Sensor Network "International Journal of Emerging Technology and Advanced Engineering Website, Volume 3, Issue 9, Pp $123-126$

[14] C. Divya (2015), “Analysis of GFEAR Protocol "International Journal of Emerging Research in Management \&Technology, Volume-4, Issue-5, Pp 38-42

[15] Parvathi (2014), “ Existing Routing Protocols for Wireless Sensor Network - A study" International Journal of Computational Engineering Research (IJCER) ,Vol, 04 ,Issue, 7 ,Pp 8-27

[16] Dr.R.KalaiMagal(2014), "A survey on wireless sensor network protocols" , ISET - International Journal of Innovative Science, Engineering \& Technology, Vol. 1 Issue 6,Pp 548-562

[17] K.Gandhimathi(2014), "A Survey on Energy Efficient Routing Protocol in Wireless Sensor Network", International Journal of Advance Research Computer Science and Management Studies, Vol 2,issue 8,Pp 174 181

[18] Rajashree.V.Biradar, "Classification and comparison of routing protocols in wireless sensor networks "ubicc journal - Volume 4,Pp 704-711

[19] Rajesh Chaudhary(2014), "A tutorial of routing protocols in wireless sensor networks" IJCSMC, vol. 3, issue. 6, pp. $971-979$

[20] Manal Abdullah(2014), “ Routing Protocols for Wireless Sensor Networks: Classifications and Challenges" Quest Journals Journal of Electronics and Communication Engineering Research Volume 2, Issue 2 ,pp: 05-15

[21] Kemal Akkaya(2005), "] A survey on routing protocols for wireless sensor networks " Elsevier, Ad Hoc Networks, Pp 325-34

[22] N. NARASIMHA DATTA(2006), ,"A survey of routing algorithms for wireless sensor networks" J. Indian Inst. Sci., 86, Pp 569-598

[23] Jay G. Shah(2015), "Technical Review of Wireless Sensor Network and its Protocol "international journal for research in emerging science and technology, Volume-2, ISSUE-1,Pp 35-41

[24] Shital Y. Agrawal(2013), "A Survey on Location Based Routing Protocols for Wireless Sensor Network "International Journal of Emerging Technology and 
Advanced Engineering Website, Volume 3, Issue 9, Pp 123-126

[25] C. Divya (2015), “Analysis of GFEAR Protocol "International Journal of Emerging Research in Management \&Technology, Volume-4, Issue-5, Pp 38-42

[26] Parvathi (2014), “ Existing Routing Protocols for Wireless Sensor Network - A study" International Journal of Computational Engineering Research (IJCER) ,Vol, 04 ,Issue, 7 ,Pp 8-27

[27] Dr.R.KalaiMagal(2014), “A survey on wireless sensor network protocols" , ISET - International Journal of Innovative Science, Engineering \& Technology, Vol. 1 Issue 6,Pp 548-562
[28] K.Gandhimathi(2014), "A Survey on Energy Efficient Routing Protocol in Wireless Sensor Network" , International Journal of Advance Research Computer Science and Management Studies, Vol 2,issue 8,Pp 174181

[29] Rajashree.V.Biradar, " Classification and comparison of routing protocols in wireless sensor networks "UbiCC Journal - Volume 4,Pp 704-711

[30] Rajesh Chaudhary(2014), "A tutorial of routing protocols in wireless sensor networks" IJCSMC, Vol. 3, Issue. 6, Pp.971 - 979 